\title{
An Explicit MOT-TD-VIE Solver for Time Varying Media
}

\author{
Sadeed B. Sayed ${ }^{1}$, H. Arda Ulku ${ }^{1,2}$, and Hakan Bagci ${ }^{1}$ \\ ${ }^{1}$ Computer, Electrical and Mathematical Sciences and Engineering \\ King Abdullah University of Science and Technology (KAUST), Thuwal, Jeddah, 23955-6900, Saudi Arabia \\ e-mails: \{sadeed.sayed, huseyin.ulku, hakan.bagci\}@kaust.edu.sa \\ ${ }^{2}$ Electronics Engineering, Gebze Technical University, Gebze, Kocaeli, 41400, Turkey, e-mail: haulku@gtu.edu.tr
}

\begin{abstract}
An explicit marching on-in-time (MOT) scheme for solving the time domain electric field integral equation enforced on volumes with time varying dielectric permittivity is proposed. Unknowns of the integral equation and the constitutive relation, i.e., flux density and field intensity, are discretized using full and half Schaubert-Wilton-Glisson functions in space. Temporal interpolation is carried out using band limited approximate prolate spherical wave functions. The discretized coupled system of integral equation and constitutive relation is integrated in time using a $P E(C E)^{m}$ type linear multistep scheme. Unlike the existing MOT methods, the resulting explicit MOT scheme allows for straightforward incorporation of the time variation in the dielectric permittivity.
\end{abstract}

\section{INTRODUCTION}

Recent advancements in material synthesis has allowed for development of electromagnetic and optical systems where the dielectric permittivity can be controlled externally, resulting in time varying media. Among the several techniques developed for analyzing transient electromagnetic field interactions on scatterers with time-dependent material properties, time domain volume integral equation (TD-VIE) solvers offer several advantages over their differential equation counterparts [1].

Recently, a stable and accurate explicit marching on-in-time (MOT) scheme has been developed for solving time domain magnetic field surface integral equation (TD-MFSIE) [2] and electric field volume integral equation (TD-EFVIE) [3]. In this work, this explicit MOT solver is extended for analyzing transient electromagnetic field interactions on scatterers with time-dependent material properties. This extension discretizes the TD-EFVIE and (time-varying) constitutive relation separately [1], [4], [5]. The unknowns flux density and field intensity are approximated in terms of full and half SchaubertWilton-Glisson (SWG) functions in space [5]. Temporal interpolation required for accurate computation of retarded time integrals carried out using approximate prolate spherical wave (APSW) functions [6]. The resulting coupled system of equations is integrated in time using a $P E(C E)^{m}$ type linear multistep scheme. The stability of resulting explicit MOT scheme is ensured using the complex-exponent extrapolation scheme developed in [7]. Unlike the existing MOT methods, explicitness of the marching scheme allows for straightforward incorporation of the time-dependent dielectric permittvitiy.

\section{FORMULATION}

Let $V$ denote the volume of a scatterer with time-dependent dielectric permittivity residing in an unbounded background medium. The permittivity and permeability of the scatterer and background are $\left\{\varepsilon(\mathbf{r}, t), \mu_{0}\right\}$ and $\left\{\varepsilon_{0}, \mu_{0}\right\}$, respectively. The scatter is excited by a band-limited incident electric field $\mathbf{E}^{\text {inc }}(\mathbf{r}, t)$. Upon excitation, flux density $\mathbf{D}(\mathbf{r}, t)$ and field intensity $\mathbf{E}(\mathbf{r}, t)$ are induced in $V$. They satisfy

$$
\begin{gathered}
\mathbf{D}(\mathbf{r}, t)=\varepsilon(\mathbf{r}, t) \mathbf{E}(\mathbf{r}, t) \\
\partial_{t} \mathbf{E}^{\mathrm{inc}}(\mathbf{r}, t)=\partial_{t} \mathbf{E}(\mathbf{r}, t)+\mathcal{L}\{\mathbf{E}(\mathbf{r}, t)\}-\mathcal{L}\left\{\varepsilon_{0}^{-1} \mathbf{D}(\mathbf{r}, t)\right\}
\end{gathered}
$$

for $\mathbf{r} \in V$. Here, $\mathcal{L}\{$.$\} is the EFVIE operator [4]. To numeri-$ cally solve (1) and (2), $V$ is discretized into tetrahedrons and unknowns $\mathbf{D}(\mathbf{r}, t), \mathbf{E}(\mathbf{r}, t)$, and $\partial_{t} \mathbf{E}(\mathbf{r}, t)$ are expanded as

$$
\begin{aligned}
\mathbf{D}(\mathbf{r}, t) & =\sum_{n=1}^{N_{\mathrm{D}}}\{\mathbf{I}(t)\}_{n} \mathbf{f}_{n}^{\mathrm{D}}(\mathbf{r}) \\
\mathbf{E}(\mathbf{r}, t) & =\sum_{n=1}^{N_{\mathrm{E}}}\{\mathbf{L}(t)\}_{n} \mathbf{f}_{n}^{\mathrm{E}}(\mathbf{r}) \\
\partial_{t} \mathbf{E}(\mathbf{r}, t) & =\sum_{n=1}^{N_{\mathrm{E}}}\{\mathbf{K}(t)\}_{n} \mathbf{f}_{n}^{\mathrm{E}}(\mathbf{r}) .
\end{aligned}
$$

Here, $\left\{\mathbf{f}_{n}^{\mathrm{D}}(\mathbf{r}), \mathbf{f}_{n}^{\mathrm{E}}(\mathbf{r})\right\}$ are the full and half SWG functions respectively [5], and $\{\mathbf{I}(t), \mathbf{L}(t), \mathbf{K}(t)\}_{n}$ are unknown timedependent expansion coefficients. First, (3) is inserted into (1) and (2), then the resulting equations are tested by $\mathbf{f}_{n}^{\mathrm{D}}(\mathbf{r}), m=$ $1: N^{\mathrm{D}}$, and $\mathbf{f}_{n}^{\mathrm{E}}(\mathbf{r}), m=1: N^{\mathrm{E}}$, respectively, at times $j \Delta t$. To facilitate the computation of retarded-time integrals, bandlimited APSW functions are applied to temporal samples $\mathbf{I}_{i}=$ $\mathbf{I}(i \Delta t), \mathbf{L}_{i}=\mathbf{L}(i \Delta t)$, and $\mathbf{K}_{i}=\mathbf{K}(i \Delta t)$. This discretization procedure yields:

$$
\begin{gathered}
\mathbf{G}^{\mathrm{DD}} \mathbf{I}_{j}=\mathbf{G}^{\mathrm{DE}} \varepsilon_{j} \mathbf{L}_{j} \\
\mathbf{G}^{\mathrm{EE}} \mathbf{K}_{j}=\mathbf{Z}_{0}^{\mathrm{EE}} \mathbf{L}_{j}-\mathbf{Z}_{0}^{\mathrm{ED}} \mathbf{I}_{j}+\mathbf{V}_{j}^{\mathrm{inc}}+\sum_{i=1}^{j-1} \mathbf{Z}_{j-i}^{\mathrm{EE}} \mathbf{L}_{i} \\
-\sum_{i=1}^{j-1} \mathbf{Z}_{j-i}^{\mathrm{ED}} \mathbf{I}_{i} .
\end{gathered}
$$

Here, elements of the matrices $\mathbf{G}^{\mathrm{DD}}, \mathbf{G}^{\mathrm{DE}}, \mathbf{G}^{\mathrm{EE}}, \boldsymbol{\varepsilon}_{j}, \mathbf{Z}_{j-i}^{\mathrm{EE}}$, and $\mathbf{Z}_{j-i}^{\mathrm{ED}}$ and the vector $\mathbf{V}_{j}^{\text {inc }}$ are

$$
\begin{aligned}
\left\{\mathbf{G}^{\mathrm{PP}}\right\}_{m, n} & =\int_{V_{m}} \mathbf{f}_{m}^{\mathrm{P}}(\mathbf{r}) \cdot \mathbf{f}_{n}^{\mathrm{P}}(\mathbf{r}) d \mathbf{r}, \mathrm{P} \in\{\mathrm{E}, \mathrm{D}\} \\
\left\{\varepsilon_{j}\right\}_{n, n} & =\left.\varepsilon\left(\mathbf{r}_{n}, t\right)\right|_{t=j \Delta t}
\end{aligned}
$$




$$
\begin{aligned}
\left\{\mathbf{Z}_{j-i}^{\mathrm{EE}}\right\}_{m, n} & =\left.\int_{V_{m}} \mathbf{f}_{m}^{\mathrm{E}}(\mathbf{r}) \cdot \mathcal{L}\left\{\mathbf{f}_{n}^{\mathrm{E}}(\mathbf{r}) T(t-i \Delta t)\right\}\right|_{t=j \Delta t} d \mathbf{r} \\
\left\{\mathbf{Z}_{j-i}^{\mathrm{ED}}\right\}_{m, n} & =\left.\int_{V_{m}} \mathbf{f}_{m}^{\mathrm{E}}(\mathbf{r}) \cdot \mathcal{L}\left\{\varepsilon_{0}^{-1} \mathbf{f}_{n}^{\mathrm{D}}(\mathbf{r}) T(t-i \Delta t)\right\}\right|_{t=j \Delta t} d \mathbf{r} \\
\left\{\mathbf{V}_{j}^{\mathrm{inc}}\right\}_{m} & =\left.\int_{V_{m}} \mathbf{f}_{m}^{\mathrm{E}}(\mathbf{r}) \cdot \partial_{t} \mathbf{E}^{\mathrm{inc}}(\mathbf{r}, t)\right|_{t=j \Delta t} d \mathbf{r} .
\end{aligned}
$$

Coupled system of equations (4) and (5) is integrated in time using a linear $k$-step $P E(C E)^{m}$ scheme to find unknown coefficients $\mathbf{I}_{j}, \mathbf{L}_{j}$, and $\mathbf{K}_{j}$. Accuracy and stability of the predictor-corrector steps are controlled using successive over relaxation (SOR) [3]. The resulting MOT scheme can be written as:

At time step $j\left(j=1: N_{\mathrm{t}}\right)$

Step 0: Compute the part of the right hand side that does not change within a time step:

$$
\mathbf{V}_{j}^{\text {fixed }}=\mathbf{V}_{j}^{\mathrm{inc}}+\sum_{i=1}^{j-1} \mathbf{Z}_{j-i}^{\mathrm{EE}} \mathbf{L}_{i}-\sum_{i=1}^{j-1} \mathbf{Z}_{j-i}^{\mathrm{ED}} \mathbf{I}_{i}
$$

Step 1: Predict $\mathbf{L}_{j}^{(0)}$ (using the predictor coefficients $\mathbf{p}$ ):

$$
\mathbf{L}_{j}^{(0)}=\sum_{l=1}^{k}\left[\{\mathbf{p}\}_{l} \mathbf{L}_{j-1+l-k}+\{\mathbf{p}\}_{k+l} \mathbf{K}_{j-1+l-k}\right]
$$

Step 2: Compute $\varepsilon_{j}$ and evaluate $\mathbf{I}_{j}^{(0)}$ by solving

$$
\mathbf{G}^{\mathrm{DD}} \mathbf{I}_{j}^{(0)}=\mathbf{G}^{\mathrm{DE}} \varepsilon_{j} \mathbf{L}_{j}^{(0)}
$$

Step 3: Evaluate $\mathbf{K}_{j}^{(0)}$ by solving

$$
\mathbf{G}^{\mathrm{EE}} \mathbf{K}_{j}^{(0)}=\mathbf{Z}_{0}^{\mathrm{EE}} \mathbf{L}_{j}^{(0)}-\mathbf{Z}_{0}^{\mathrm{ED}} \mathbf{I}_{j}^{(0)}+\mathbf{V}_{j}^{\text {fixed }}
$$

Step 4: Iterate until the solution converges $\left(\nu=1: \nu_{\max }\right)$

Step 4.1: Correct $\mathbf{L}_{j}^{(\nu)}$ (using corrector coefficients $\mathbf{c}$ ):

$$
\begin{aligned}
\mathbf{L}_{j}^{(\nu)} & =\sum_{l=1}^{k}\left[\{\mathbf{c}\}_{l} \mathbf{L}_{j-1+l-k}\right. \\
& \left.+\{\mathbf{c}\}_{k+l} \mathbf{K}_{j-1+l-k}\right]+\{\mathbf{c}\}_{2 k+1} \mathbf{K}_{j}^{(\nu-1)}
\end{aligned}
$$

Step 4.2: Apply SOR:

$$
\mathbf{L}_{j}^{(\nu)}=\alpha \mathbf{L}_{j}^{(\nu)}+(1-\alpha) \mathbf{L}_{j}^{(\nu-1)}
$$

Step 4.3: Compute $\varepsilon_{j}$ and evaluate $\mathbf{I}_{j}^{(\nu)}$ by solving

$$
\mathbf{G}^{\mathrm{DD}} \mathbf{I}_{j}^{(\nu)}=\mathbf{G}^{\mathrm{DE}} \varepsilon_{j} \mathbf{L}_{j}^{(\nu)}
$$

Step 4.4: Evaluate $\mathbf{K}_{j}^{(\nu)}$ by solving

$$
\mathbf{G}^{\mathrm{EE}} \mathbf{K}_{j}^{(\nu)}=\mathbf{Z}_{0}^{\mathrm{EE}} \mathbf{L}_{j}^{(\nu)}-\mathbf{Z}_{0}^{\mathrm{ED}} \mathbf{I}_{j}^{(\nu)}+\mathbf{V}_{j}^{\mathrm{fixed}}
$$

Step 4.5: Check for the convergence. If the solution converged, set $\mathbf{L}_{j}=\mathbf{L}_{j}^{(\nu)}, \mathbf{K}_{j}=\mathbf{K}_{j}^{(\nu)}$ and $\mathbf{I}_{j}=\mathbf{I}_{j}^{(\nu)}$

It should be noted here that $\mathbf{G}^{\mathrm{DD}}, \mathbf{G}^{\mathrm{DE}}$, and $\mathbf{G}^{\mathrm{EE}}$ are sparse, therefore (8), (9), (12), and (13) can be very efficiently solved using an iterative solver.

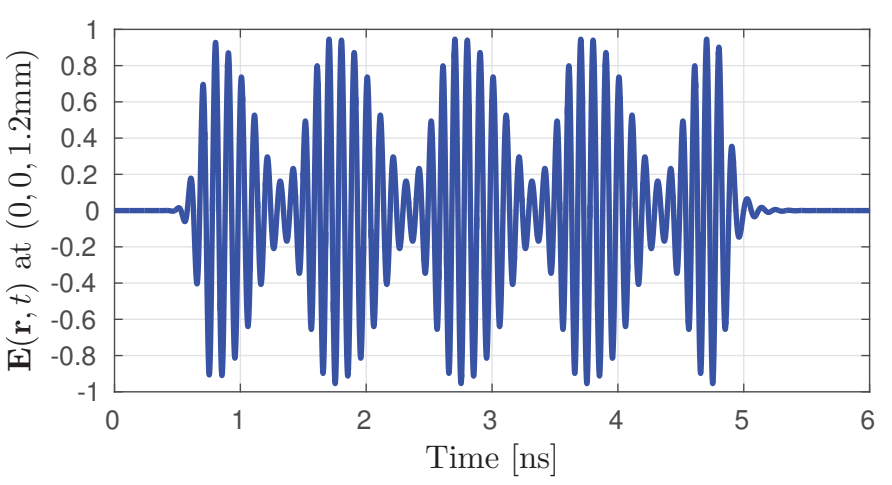

Fig. 1. $\mathbf{E}(\mathbf{r}, t)$ recorded at $(0,0,1.2 \mathrm{~mm})$

\section{Numerical Results}

The proposed solver is used for analyzing transient electromagnetic field interactions on a dielectric slab with dimensions $90 \times 90 \times 3 \mathrm{~mm}^{3}$ and $\varepsilon(\mathbf{r}, t)=3 \varepsilon_{0}\left(1+b \sin \left(2 \pi f_{\mathrm{m}} t\right)\right)$, $f_{\mathrm{m}}=1 \mathrm{GHz}$, and $b=0.37$. The slab is excited by $\mathbf{E}^{\text {inc }}(\mathbf{r}, t)=\hat{\mathbf{x}} G\left(t-\mathbf{r} \cdot \hat{\mathbf{z}} / c_{0}\right)$, where

$$
G(\mathbf{r}, t)=\left\{\begin{array}{l}
\cos \left[2 \pi f_{0}\left(t-t_{1}\right)\right] e^{-\left(t-t_{0}\right)^{2} / 2 \sigma^{2}}, t<t_{1} \\
\cos \left[2 \pi f_{0}\left(t-t_{1}\right)\right], t_{1} \leq t<t_{2} \\
\cos \left[2 \pi f_{0}\left(t-t_{2}\right)\right] e^{-\left(t-t_{2}\right)^{2} / 2 \sigma^{2}}, t_{2} \leq t
\end{array}\right.
$$

with $f_{0}=10 \mathrm{GHz}, \sigma=3 /\left(\pi f_{0}\right), t_{1}=7 \sigma$, and $t_{2}=$ $t_{1}+20 /\left(\pi f_{0}\right)$. For this simulation, $N^{\mathrm{E}}=37652, N^{\mathrm{D}}=$ $21200, \Delta t=12 \mathrm{ps}, N_{t}=5600, \alpha=0.3, \mathbf{p}$ and $\mathbf{c}$ are the sixth order Adams-Bashforth and backward differentiation (BDF) coefficients, and the convergence threshold at Step 4 is $10^{-13}$. The stability of the solution is ensured using the complex-exponent extrapolation scheme developed in [7] for high contrast scatterers. Fig. 1 shows $\mathbf{E}(\mathbf{r}, t)$ recorded at $\mathbf{r}=(0,0,1.2) \mathrm{mm}$. The result clearly shows that the time variation in $\varepsilon(\mathbf{r}, t)$ results in amplitude modulation in $\mathbf{E}(\mathbf{r}, t)$.

\section{REFERENCES}

[1] G. Kaur and A. E. Yilmaz, "ET-AIM accelerated analysis of scattering from inhomogeneous objects with time-varying permittivity," in Proc. USNC-URSI, p. 113, July 2015.

[2] H. A. Ulku, H. Bagci, and E. Michielssen, "Marching on-in-time solution of the time domain magnetic field integral equation using a predictorcorrector scheme," IEEE Trans. Antennas Propag., vol. 61, no. 8, pp. 4120-4131, Aug. 2013.

[3] S. B. Sayed, H. A. Ulku, and H. Bagci, "Stable quasi-explicit MOT solver for the time domain volume electric field integral equation," in Proc. Appl. Comput. Electrom. Symp, pp. 416-420, Mar. 2014.

[4] H. A. Ulku, S. B Sayed, and H. Bagci, "An explicit MOT-TDVIE scheme for analyzing electromagnetic field interactions on nonlinear scatterers," in Proc. ICCEM, pp. 101-103, Feb. 2015.

[5] G. Kobidze, J. Gao, S. Balasubramaniam, and E. Michielssen, "A fast time domain integral equation based scheme for analyzing scattering from dispersive objects," IEEE Trans. Antennas Propag., vol. 53, no. 3, pp. 1215-1226, Mar. 2005.

[6] R. Wildman, G. Pisharody, D. S. Weile, S. Balasubramaniam, and E. Michielssen, "An accurate scheme for the solution of the time-domain integral equations of electromagnetics using higher order vector bases and bandlimited extrapolation," IEEE Trans. Antennas Propag., vol. 52, no. 11, pp. 2973-2984, Nov. 2004.

[7] S. B. Sayed, H. A. Ulku, and H. Bagci, "A stable marching on-in-time scheme for solving the time domain electric field volume integral equation on high-contrast scatterers," IEEE Trans. Antennas Propag., vol. 63, no. 7, pp. 3098-3110, July 2015. 